\title{
Environmental Conditions-based Analysis of Energy Harvesting Systems for Wireless Sensor Networks
}

\author{
Scott C. Kempf ${ }^{1}$, Frank Schäfer ${ }^{1}$ \\ ${ }^{1}$ Fraunhofer Institute for High-Speed Dynamics, Ernst-Mach-Institut, 79104 Freiburg, Germany \\ scott.kempf@emi.fraunhofer.de
}

\begin{abstract}
:
The increasing efficiency of ultra-low-power electronic components and the growing market of energy harvesters have together led to an increasing number of self-sufficient energy harvesting applications in a wide array of fields. The goal of this work is the development of a methodology for determining the amount of ambient and harvestable energy available in various environments. The developed methodology can be used to help facilitate the design of energy autarkic systems utilizing energy harvesting techniques.
\end{abstract}

This paper describes the methodology and an example application, which takes the form of an energy autarkic wireless sensor network applied in a typical office environment.

Key words: energy harvesting, wireless sensor networks, self-sufficient, analysis methodology.

\section{Introduction}

Due to the increasing efficiency and availability of energy harvesters and energy harvesting technologies, as well as the advances in ultralow power (ULP) energy consumers, interest in energy harvesting is increasing and its field of application is growing [1], [2]. Additionally, many environments offer various ambient energy sources which, through hybrid energy harvesting systems, can expand the potential field of application.

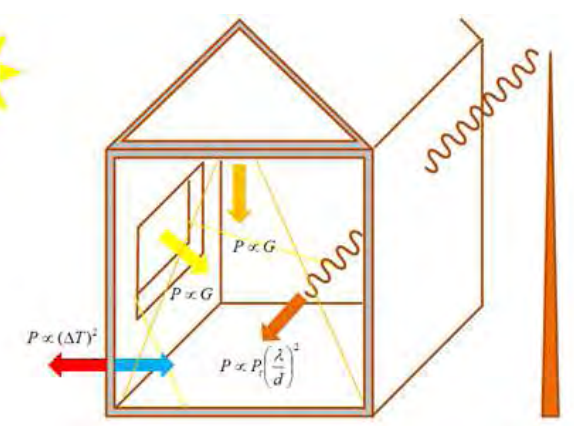

Fig. 1. Energy harvesting opportunities in typical office or home environment.

Figure 1 demonstrates potential ambient energy sources in a typical office or home environment. Depending on the available irradiation, $G$ $\left[\mathrm{W} / \mathrm{m}^{2}\right]$, solar cells provide significant energy harvesting gains via the photovoltaic effect. Outdoor temperature fluctuations provide energy harvesting gains through thermoelectric generators using the temperature differentials,
$\Delta T \quad\left[{ }^{\circ} \mathrm{C}\right]$, between inner and outer spaces. Electromagnetic radiation from local high frequency or tower-based transmitters can provide small amounts of energy via induction.

A typical energy harvesting system includes both an energy harvester and energy storage. The stored and harvested energies are delivered via an energy management system to low-power sensors, a microcontroller and an RF transceiver. The microcontroller reads in sensor input and uses the transceiver to wirelessly transmit the collected data to a central system. This example system is visualized in Figure 2.

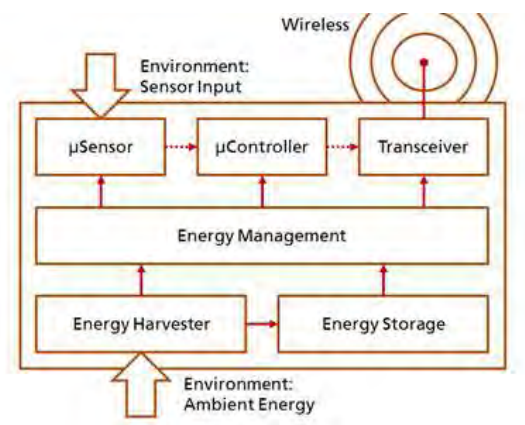

Fig. 2. Wireless sensor node employing energy harvesting.

In order to determine the feasibility and configuration for the design of an energy harvesting system, an energy balance is required, which is dependent on an accurate forecasts and analyses of ambient energy sources and conversion efficiencies of the components in the energy chain between 
harvester and consumer. The execution of an accurate analysis is highly environment and application specific.

A general overview of energy harvesting, including energy harvesting in the context of the greater energy and power electronics fields, a discussion and comparison of established and emerging technologies on the basis of physical principle, implementation of technologies, and potential gains, can be found in various scientific papers, presentations and reports [1][3]. Specific energy harvesting projects are also documented, which can provide insight into designs for developing similar projects [4],[5]. The physical principles involved in the various energy harvesting techniques are discussed in both text books and scientific papers [5]-[7], although they often require supplemental information regarding environmental conditions and devices characteristics. Device specific data sheets provide specific harvesting and power conversion devices, which are applied to energy balance calculations [8]-[11]. However, despite a wealth of information over various sources, there is currently no comprehensive documentation to enable developers the depth and flexibility required for a complete analysis of the entire energy harvesting chain, including the characterization and calculation of individual harvesting sources.

By developing a methodology, the analysis of potential energy harvesting applications is simplified. This has been completed through the synthesis of state-of-the-art analyses of the various energy sources and commercially available energy harvesters, ULP consumers and high efficiency energy storage devices. The methodology presented is formula-based and is meant to be applied during the initial project management stages.

Through the development of the methodology, he following three physical principles where analyzed:

- the photovoltaic effect,

- the thermoelectric effect, and

- electromagnetic induction (RF).

For each physical principle, information is provided for the modeling of available ambient energy, environmental- and harvester-based conversion efficiencies, and harvester outputbased adaptation circuit efficiencies.

In order to provide the most critical details of the analysis model, the description of these individual models have been omitted.

\section{Development of the Methodology}

The analysis of potential energy harvesting applications is performed by modeling an energy chain, as seen in figure 3 .

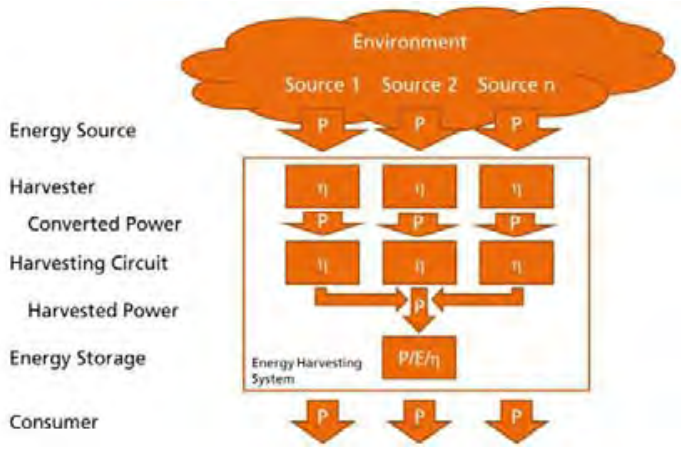

Fig. 3. Energy conversion chain with characteristic values.

The methodology is broken down into the analysis of potential energy sources and consumer energy demand and the definition minimum energy storage characteristics for the successful matching of generation and consumption profiles. The results of generation and consumption profiles are given in terms of power as a function of time, as well as peak and average power and total energy over a defined harvesting cycle. The analysis of individual sources is further broken down to available ambient power or energy, and the efficiencies of both the conversion from ambient to electrical energy and the adaptation of the harvested electricity to the storage system.

\section{Energy Sources and Conversion}

The analysis of available ambient energy and conversion and harvesting circuit efficiencies varies by physical principle. The output of each harvesting circuit is dependent on environmental conditions as well as the characteristics of other devices in the conversion chain. After application of losses at each conversion step, harvested energy can be given in terms of generated power $P_{g, s}(t)$ as a function of time, where $s$ is the source index.

Combining multiple power sources into total generated power $P_{g}(t)$ is achieved by summing the individual power sources.

For the purposes of loading energy storage or ensuring the continuous supply of sufficient energy, peak power $P_{g}^{\text {peak }}(1)$, average power $\bar{P}_{g}(2)$ and total energy $E_{g}^{x}$ (3) generation are calculated ( $i=$ time step index, $n=$ number of time steps in an harvesting cycle, $x=$ length of harvesting cycle). Assuming discrete time steps:

$P_{g}^{\text {peak }}=\max _{1 \leq i \leq n} P_{g}\left(t_{i}\right)$ 
$\overline{P_{g}}=\frac{\sum_{i=1}^{n} P_{g}\left(t_{i}\right)}{n}$

$E_{g}^{x}=\overline{P_{g}} \cdot x$

The resultant characterization of the energy harvesting system provides the generation phase of the energy chain.

\section{Energy Storage}

The energy storage system must be able to provide two functions: the bridging of generation gaps in the harvesting cycle with sufficient energy for continuous operation of the consumer (long-term storage) and the provision of sufficient power during short wake-up periods (short-term storage). When considering energy storage options, there is also a trade-off between energy density and power density. Batteries typically provide the long-term storage functionality due to high energy densities and low power densities, whereby capacitors, or supercapacitors, provide large amounts of power for the short-term demands, over short periods of time.

In addition to higher energy densities, batteries provide an advantage over super capacitors in that they tend to hold their nominal voltage outputs over a larger charge range. The following characteristics are relevant when discussing secondary cell batteries: nominal capacity, nominal voltage, nominal charging/discharging current, efficiency, selfdischarge rate [\%/year], charge-discharge cycle lifespan, charge procedure and cost [€/Wh].

While supercapacitors suffer from increased self-discharge when compared with ceramic capacitors, the latter rarely provide sufficient energy capacity for the short-term energy storage requirements, therefor supercapacitors are most often applied in the energy storage system.

\section{Analysis model}

The requirements of the storage system (st) are dependent on the results of power generation $(g)$ and consumption $(c)$ analyses. The initial calculation regards the minimum total energy storage system efficiency $\eta_{s t}^{\min }\left(\bar{P}_{c}=\right.$ average consumer demand, $\bar{P}_{g}=$ average harvester generation) (4).

$\eta_{s t}^{\min }=\frac{\overline{P_{c}}}{\overline{P_{g}}}$

The long-term storage or battery $(B)$ component is characterized considering a long-term cyclical period. The long-term storage component of the energy storage system has to provide enough energy to bridge gaps in generation periods. The minimum deliverable energy $\Delta E_{B}^{\min }$ is determined by comparing average generation $\bar{P}_{g}$ and demand $\overline{P_{c}}$ over periods of under generation $\left(\overline{P_{c}}>\bar{P}_{g}\right) t_{0}$ to $t_{n}\left(\Delta t_{i}=\right.$ duration of time step, $i=$ time step index, $n=$ number of time steps in under generation period (5).

$\Delta E_{B}^{\min }=\sum_{i=0}^{n}\left(\bar{P}_{c}\left(t_{i}\right)-\bar{P}_{g}\left(t_{i}\right)\right) \Delta t_{i}$

This characterization of the required energy is conservative, in that it assumes that all required energy over the course of one cycle will be needed during one continuous period. It is possible that multiple distinct charging and discharging periods exist, thereby decreasing long-term energy storage requirements.

Because batteries are typically characterized by nominal voltage and charge, the total deliverable charge $\Delta q_{B}^{\min }$ must be calculated assuming the constant (nominal) voltage $U_{B}$ over the charge range $q_{l}$ to $q_{u}(6)$.

$\Delta E_{B}^{\min }=\int_{q_{l}}^{q_{u}} U(q) \cdot d q$

$\rightarrow \Delta q_{B}^{\min }=q_{u}-q_{l}=\frac{\Delta E_{B}^{\min }}{U_{B}}$

To determine the total required charge capacity $q_{B}^{\min }$ of the battery, either the depth of charge (DOC) or the lower charge limit, $q_{B, l}$ should be considered (7).

$q_{B}^{\min }=\frac{100}{D O C} \cdot \Delta q_{B}^{\min }=q_{B, l}+\Delta q_{B}^{\min }$

The minimum required charging $P_{B, c h}^{\min }$ (8) and discharging $P_{B \text {,disch. }}^{\min }$ (9) powers are also necessary for characterization of the long term energy storage. The charging limit is dependent on the total energy discharged $\Delta E_{B}^{\min }\left(P_{c}>P_{g}\right)$ over a cyclical period and the total charging time $t_{B, c h} .\left(P_{g}>P_{c}\right)$. This assumes that the available charging power exceeds the calculated minimum charging capacity. If this is not the case, the minimum charging current must be increased to accommodate a shorter charging period. The minimum discharging power is not limited in this way, because demand spikes are accommodated by the short-term energy storage.

$P_{B, \text { ch. }}^{\text {min }}=\frac{\Delta E_{B}^{\text {min }}}{t_{B, c h .}}$
$P_{B, \text { disch. }}^{\text {min }}=\overline{P_{C}}$

As before, the minimum power $P^{\min }$ can be converted to current $I^{\mathrm{min}}$ (10) using the simplified (nominal) battery voltage, $U_{B}$.

$I_{B, \text { ch. }}^{\min }$ disch. $=\frac{P_{B, \text { ch./disch. }}^{\min }}{U_{B}}$

The short-term energy storage must be characterized differently than battery storage. 
The minimum output capacitance $C_{S C}$ (12) for the short-term storage system (supercapacitor) is derived from the characteristic capacitor energy equation (11) $\left(E_{\text {stored }}=\right.$ stored energy, $U_{u}$ and $U_{l}=$ upper and lower system voltage limits, respectively; $E_{c, j}=$ total energy required for the consumer job (see section on consumer analysis)).

$$
\begin{aligned}
& E_{\text {stored }}=\frac{1}{2} C U^{2} \\
& \rightarrow E_{C, j}=\Delta E_{\text {stored }}=\frac{1}{2} C\left(U_{u}^{2}-U_{l}^{2}\right) \\
& \rightarrow C_{S C}=\frac{2 \cdot E_{c, j}}{U_{u}^{2}-U_{l}^{2}}
\end{aligned}
$$

\section{Consumer}

A typical consumer application for energy harvesting systems is the wireless sensor network, a node of which generally consists of a microcontroller, an RF transceiver and sensing hardware. The most important strategies for saving power are limiting time spent in active mode and eliminating power consuming functionality in low-power mode. Considering this, the consumer analysis is performed by generating a consumer profile. This profile is modeled as base power consumption with specific cyclical jobs. Each job is broken up into specific phases (See Figure 4).

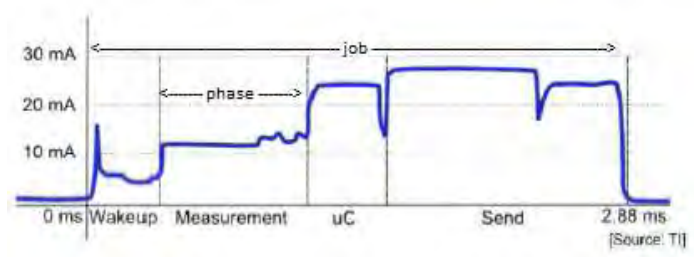

Fig. 4. Example current consumption profile for energy harvesting application with job phases. [3]

Individual consumer phases $(p h)$ are defined by power, or current, consumption and duration. In the case of voltage drops due to capacitor discharge, an average voltage can be assumed $\left(E_{p h}=\right.$ phase energy consumption, $P_{p h}=$ average phase power consumption, $t_{p h}=$ phase duration) (13).

$E_{p h}=P_{p h} \cdot t_{p h}\left(P_{p h}=U_{a v e} \cdot I_{p h}\right)$

Jobs $(j)$ are defined as a collection of phases. For a specific job, peak power consumption $P_{j}^{\text {peak }}(15)$, average power consumption $\bar{P}_{j}(16)$ and total energy consumption $E_{j}$ (14) are calculated $(p h=$ job phase index, $m=$ job phase count, $t_{j}=$ total job cycle time).

$$
\begin{aligned}
& E_{j}=\sum_{p h=1}^{m} E_{p h} \\
& P_{j}^{\text {peak }}=\max _{1 \leq p h \leq m} P_{p h}
\end{aligned}
$$

$\bar{P}_{J}=\frac{E_{j}}{t_{j}}$

Using the characteristics of the individual jobs and the base power consumption, peak $P_{c}^{\text {peak }}$ (17) (assuming no overlapping jobs) and average $\bar{P}_{c}$ (18) consumer power demand as well as total consumer energy consumption $E_{c}^{x}$ (19) during a period, $x$ are determined $(j=$ consumer job index, $n=$ consumer job count).

$$
\begin{aligned}
& P_{c}^{\text {peak }}=P_{c}^{\text {base }}+\max _{1 \leq j \leq n} P_{j}^{\text {peak }} \\
& \bar{P}_{c}=P_{c}^{\text {base }}+\sum_{j=1}^{n} \bar{P}_{J} \\
& E_{c}^{x}=\bar{P}_{c} \cdot x
\end{aligned}
$$

The peak and average power provide the necessary characterization for the consumption phase of the energy chain.

\section{Example application}

The example application is a typical office space in Freiburg, Germany, on an average summer day. The environmental conditions include indirect sunlight during daylight hours, a television transmission tower at a distance of approximately $4 \mathrm{~km}$ and an oscillating temperature of between $14^{\circ} \mathrm{C}$ and $27^{\circ} \mathrm{C}$. The consumer application was defined as a wireless sensor node communicating with a central data collection server. The wireless sensor node is equipped with an ADC measurement circuit as well as temperature, humidity, pressure and movement sensors. The nominal voltage for the consumer is $3.3 \mathrm{~V}$.

\section{Source Profile}

The source profile was based on hourly conditions updates over the course of the day.

The photovoltaic profile consists of 13 hours of full indirect sunlight $\left(G=200 \mathrm{~W} / \mathrm{m}^{2}\right)$ and two hours of indirect twilight $\left(G=150 \mathrm{~W} / \mathrm{m}^{2}\right)$. Applying an amorphous silicon cell [8] with a surface area of $27.6 \mathrm{~cm}^{2}$ to the model adapted from [7], a conversion efficiency of $8.8 \%$ was determined. A harvesting circuit efficiency of $65 \%$ was applied based on expected solar cell output [9]. The resultant peak and average harvested powers are $31.7 \mathrm{~mW}$ and $19.2 \mathrm{~mW}$, respectively.

The thermoelectric profile was determined using a fluctuating outer temperature between $14^{\circ} \mathrm{C}$ and $27^{\circ} \mathrm{C}$ over the course of the day with a constant inner temperature of $23^{\circ} \mathrm{C}$. Using the characteristics of a suitable thermo-electric generator (TEG) [10], converted powers of up to $25.56 \mathrm{~mW}(\Delta \mathrm{T}=-9 \mathrm{~K})$ and $5.05 \mathrm{~mW}(\Delta \mathrm{T}=+4$ $\mathrm{K})$ were calculated. An average harvester circuit efficiency of $20 \%$ was applied based on expected TEG output [11]. The resultant peak 
and average harvesters powers are $5.1 \mathrm{~mW}$ and $1.7 \mathrm{~mW}$, respectively.

The electromagnetic harvesting profile assumes a tower-based transmitter at a distance of $4 \mathrm{~km}$ and transmitting at $10 \mathrm{~kW}$ at $868 \mathrm{kHz}$. Using the two-ray ground reflection propagation model described in [6], and neglecting antenna loss, a conversion power of $5.29 \mu \mathrm{W}$ at the reception antenna was calculated. A conservative harvesting circuit efficiency of $18 \%$ was applied based on [5]. The resultant harvested power is $0.95 \mu \mathrm{W}$.

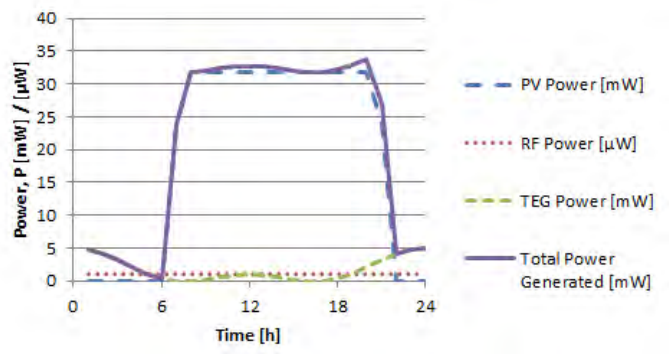

Fig. 5. Total generated power for example application with individual harvesting components

Tab. 1: Source characteristics for the example application

\begin{tabular}{|l|r|r|r|}
\hline Source & $\begin{array}{r}P_{g}^{\text {peak }} \\
\mathrm{mW}\end{array}$ & $\begin{array}{r}\bar{P}_{g} / \\
\mathrm{mW}\end{array}$ & $\begin{array}{c}E_{g}^{24 h} / \\
\mathrm{mWh}^{1}\end{array}$ \\
\hline Photovoltaic & 31.7 & 19.2 & 460.1 \\
\hline Thermoelectric & 5.1 & 1.7 & 41.5 \\
\hline Electromagnetic & 0.001 & 0.001 & 0.023 \\
\hline Total & 33.8 & 20.9 & 501.6 \\
\hline
\end{tabular}

By summing the inputs from the three harvester inputs, a total source profile was created, as in figure 5 and table 1.

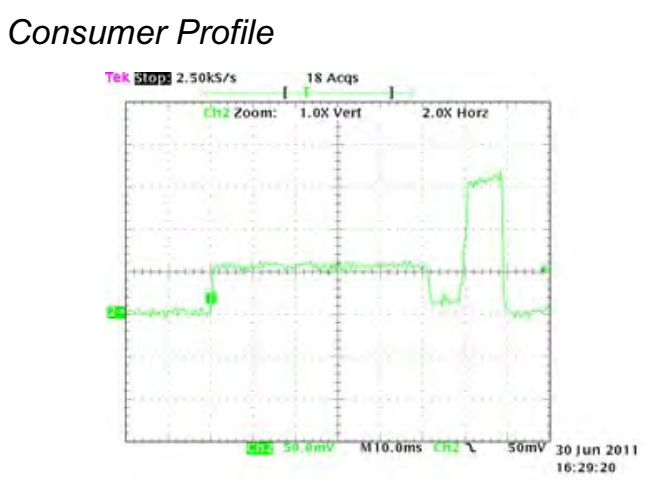

Fig. 6. Current consumption profile during active mode for the example consumer (sensor node).

A prototype consumer was built using a CC430 microcontroller from Texas Instruments and

\footnotetext{
${ }^{1}$ Depending on the time frame, energy units can be given in watt-hours or in joules, whereby $1[\mathrm{~W} \cdot \mathrm{h}]=3600[\mathrm{~J}]$.
}

several ULP sensors. Figure 6 displays the demand measurement taken over a $10 \Omega$ shunt resistor using an oscilloscope.

The first phase of the consumption profile represents the wakeup, sensor read-in and calculation phase. The second is a wait for transmission phase. Finally the peak represents the message transmission. Table 2 shows the break-down for the profile model.

Tab. 2: Phase characteristics for consumer job

\begin{tabular}{|l|l|l|l|l|}
\hline Phase & $\begin{array}{l}I_{p h} / / \\
\mathrm{mA}\end{array}$ & $\begin{array}{l}P_{p h} \quad / \\
\mathrm{mW}\end{array}$ & $\begin{array}{l}t_{p h} / \\
\mathrm{ms}\end{array}$ & $\begin{array}{l}E_{p h} \quad / \\
\mu \mathrm{J}\end{array}$ \\
\hline $\begin{array}{l}\text { 1: sensor \& } \\
\text { ADC read-in }\end{array}$ & 5.5 & 18.15 & 51 & $\sim 925$ \\
\hline 2: wait for tx & 1.5 & 4.95 & 8 & $\sim 40$ \\
\hline 3: RF tx & 16.0 & 52.80 & 10 & $\sim 528$ \\
\hline
\end{tabular}

Using these inputs, the consumer profile is viewed over the job period in figure 7 .

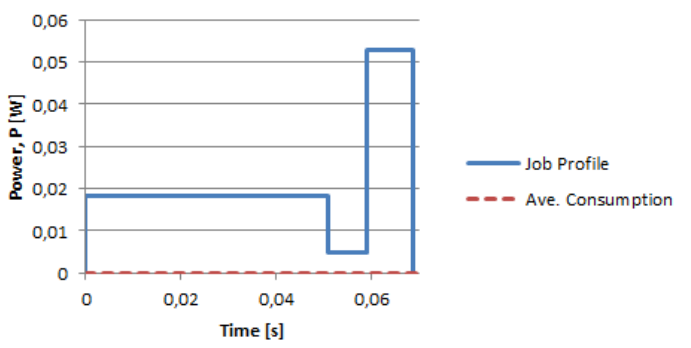

Fig. 7. Modeled example consumer job over job period $(70 \mathrm{~ms})$

By limiting the duty cycle of the consumer (cycle time is 60 seconds), it was possible to produce very low average consumption characteristics, which is shown in table 3.

Tab. 3: Consumer job characteristics

\begin{tabular}{|l|l|l|l|}
\hline Job & $\begin{array}{l}P_{j}^{\text {peak }} \\
\mathrm{mW}\end{array}$ & $\begin{array}{l}\bar{P}_{J} \\
\mathrm{~mW}\end{array}$ & $E_{j} / \mu \mathrm{J}$ \\
\hline $\begin{array}{l}\text { 1: Sensor read-in } \\
\text { and transmission }\end{array}$ & 52.80 & 0.025 & $\sim 1493$ \\
\hline
\end{tabular}

While the CC430 microcontroller itself has a typical current consumption of less than $1 \mu \mathrm{A}$ in deep-sleep mode, the inclusion of a low-power movement sensor (205 $\mu \mathrm{A}$ sleep state) increases the base demand significantly to 680 $\mu \mathrm{W}(206 \mu \mathrm{A} \cdot 3.3 \mathrm{~V})$. Table 4 demonstrates the total consumer characteristics given the base consumption ( $\mu \mathrm{C}+$ movement sensor) and the sensing and transmission job.

Tab. 4: Consumer characteristics

\begin{tabular}{|l|l|l|}
\hline$P_{c}^{\text {peak }} / \mathrm{mW}$ & $\bar{P}_{c} / \mathrm{mW}$ & $E_{c}^{24 h} / \mathrm{mWh}$ \\
\hline 53.48 & 0.705 & 16.92 \\
\hline
\end{tabular}




\section{Energy Storage}

The example application is fairly generous with regards to available source energy; both the thermoelectric and photovoltaic sources would provide more than enough energy for the consumer. In order to demonstrate the model for hybrid applications, however, they are both applied. Figure 8 compares the generation and demand curves.

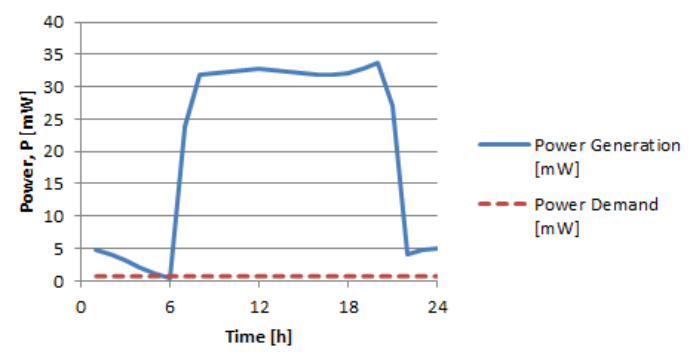

Fig. 8. Power generation vs. demand over the harvesting cycle (24h)

The final results for the energy storage system given the source and consumer profiles are given in table 5 . Due to the characterization of the selected battery, the nominal system voltage has been changed to $3.0 \mathrm{~V}$.

Tab. 5: Limit characteristics of energy storage system.

\begin{tabular}{|c|c|c|}
\hline Characteristic & Value & Comments \\
\hline$\eta_{\text {storage }}^{\min } / \%$ & 3.36 & - \\
\hline$\Delta E_{\text {bat }}^{\min } / \mu \mathrm{Wh}$ & 309.5 & $1 \mathrm{~h} @ 0.395 \mathrm{~mW}$ \\
\hline$\Delta q_{\text {bat }}^{\min } / \mu \mathrm{Ah}$ & 103.2 & @ 3 V \\
\hline$q_{\text {bat }}^{\min } / \mathrm{mAh}^{2}$ & 1.03 & $10 \%$ DOC \\
\hline$P_{\text {bat }, c h .}^{\min } / \mu \mathrm{W}$ & 13.5 & \\
\hline$I_{\text {bat }, c h .}^{\min } / \mu \mathrm{A}$ & 4.5 & @ 3 V \\
\hline$P_{\text {bat,disch. }}^{\min } / \mu \mathrm{W}$ & 705 & \\
\hline$I_{\text {bat,disch. }}^{\min } / \mu \mathrm{A}$ & 244 & @ 3 V \\
\hline$C_{\text {supercap }} / \mathrm{mF}$ & 2.57 & $\begin{array}{l}U_{u}=3.0 \mathrm{~V}, \\
U_{1}=2.8 \mathrm{~V}, \\
E_{j}=1493.26 \mu \mathrm{J}\end{array}$ \\
\hline
\end{tabular}

\section{Conclusion}

A methodology has been developed for the determination of feasibility and configuration of energy harvesting applications. The example application demonstrates the application of this methodology, including the characterization of available ambient conditions, conversion efficiencies, consumer demand and the

\footnotetext{
${ }^{2}$ Units of charge for batteries are usually given in ampere-hours, whereby 1 [A.h] = 3600 [C].
}

minimum characteristics for the energy storage system.

Analysis of the energy sources for the example application demonstrate photovoltaic and thermoelectric potential orders of magnitude higher than that available from electromagnetic sources, which brings into the question the value of investing in the developing the RF harvesting antenna and circuit for this particular application. At the same time, the photovoltaic and thermoelectric sources provide more than ample power for the demands of the conceived consumer. The minimum characteristics for the energy storage system are realistic and thereby demonstrate feasibility of the application.

\section{References}

[1] M. Raju, M. Grazier, Energy harvesting - ULP meets energy harvesting: a game-changing combination for design engineers, white paper, Texas Instruments (2010)

[2] A. Harb, Energy harvesting: State-of-the-art, Renewable Energy 36, 1-14 (2010); doi: 10.1016/j.renene.2010.06.014

[3] S. Benecke, Autarke Funksensoren - Energy Harvesting, AMA Weiterbildungsseminar, Fraunhofer IZM (2011)

[4] D. J. De Villiers, Hybrid energy harvesting system for a condition monitoring mote, Magister Thesis, Centre for Instrumentation Research, Department of Electrical Engineering, Cape Peninsula University of Technology, Cape Town.

[5] M. Mi et al., RF energy harvesting with multiple antennas in the same space, IEEE Antennas and Propagation Magazine 47:5, 100-106 (2005).

[6] Y. Huang and K. Boyle, Antennas: from theory to practice, John Wiley \& Sons Ltd, Chinchester, West Sussex, UK (2008).

[7] N. H. Reich, et al., Weak light performance and spectral response of different solar cell types, Dept. of Science, Technology and Society, Copernicus Institute for Sustainable Development and Innovation, Utrecht University, the Netherlands (2005).

[8] Amorphous solar cell - AT-7665, datasheet, Sanyo Semiconductor Co., Ltd. (2008); No.A0061.

[9] LTC3105 - 400mA step-up DC/DC converter with maximum power point control and $250 \mathrm{mV}$ startup, Linear Technology Corporation (2010); LT1010.

[10] Thermo electrical generator - TEG 241-120-15, datasheet, Thermalforce.de (2011).

[11] LTC3109 - auto-polarity, ultralow voltage step-up converter and power manager, Linear Technology Corporation (2010); LT0610. 